Comment. Math. Helv. 72 (1997) 67-71

0010-2571/97/010067-5 \$1.50+0.20/0
(C) 1997 Birkhäuser Verlag, Basel

Commentarii Mathematici Helvetici

\title{
Complete surfaces of at most quadratic area growth
}

Peter $\mathrm{Li}^{*}$

\begin{abstract}
In this article, we study complete surfaces with finite topological type and has at most quadratic area growth. In particular, we show that if the curvature of such a surface does not change sign, then it must be of finite total curvature.
\end{abstract}

Mathematics Subject Classification (1991). 53C20

Keywords. Riemannian manifold, Gaussian curvature, total curvature, finite topological type.

In 1935, Cohn-Vossen [CV] studied the validity of the Gauss-Bonnet theorem for complete non-compact surfaces. In particular, he considered the relationship between the Euler characteristic $\chi(M)$ and the integral of the Gaussian curvature $K$ of a complete surface, $M^{2}$, without boundary. He proved that if for any compact exhaustion $\left\{\Omega_{i}\right\}$ of $M$, the limit

exists, then

$$
\int_{M} K=\lim _{i \rightarrow \infty} \int_{\Omega_{i}} K
$$

$$
\int_{M} K \leq 2 \pi \chi(M)
$$

Later on, in 1957, Huber [Hu1] proved that if the negative part of the Gaussian curvature of $M$ defined by

$$
K_{-}=\max \{-K, 0\}
$$

is integrable, then

$$
\int_{M} K \leq 2 \pi \chi(M)
$$

and $M$ is conformally equivalent to a compact Riemann surface with finitely many punctures. In particular, this implies that $M$ has finite topological type. It also implies that the positive part of the Gaussian curvature $K_{+}=\max \{0, K\}$ is integrable and hence $M$ must have finite total curvature, i.e.,

* Research supported in part by NSF grant \# DMS-9626310 


$$
\int_{M}|K|<\infty
$$

In 1964, Hartman [H] farther proved that under the assumption

$$
\int_{M} K_{-}<\infty
$$

the area $A(r)$ of geodesic balls of radius $r$ at a fixed point must grow at most quadratically in $r$. Moreover, (also see $[\mathrm{S}]$ ),

$$
\int_{M} K+\lim _{r \rightarrow \infty} \frac{2 A(r)}{r^{2}}=2 \pi \chi(M) .
$$

The results of Huber and Hartman assert that the finiteness of $\int_{M} K_{-}$implies the finiteness of $\chi(M)$ and $\lim \left(A(r) / r^{2}\right)$. Moreover, (1) holds. It is then natural to ask if the converse of this statement is valid. It turns out that the answer is negative as indicated by the following example. Let us consider an arbitrary non-flat metric on a torus. Its universal Riemannian covering $M$ is given by $\mathbb{R}^{2}$ endowed with a non-flat $\mathbb{Z} \times \mathbb{Z}$ invariant metric. Clearly, $M$ has finite topological type and the area growth is quadratic because the metric is uniformly equivalent to the flat metric. However, the total curvature and also the integral $\int_{M} K_{-}$are not finite because of periodicity of the metric.

The purpose of this note is to prove that the finiteness of $\chi(M)$ and $\lim \left(A(r) / r^{2}\right)$ together imply the finiteness of the total curvature if we assume that the Gaussian curvature of the surface is of one sign at each end.

We would like to remark that surfaces with finite total curvature have been extensively studied by [Hu2], [F], and [L-T]. Using a different argument, Chen [C] independently proved that if $M$ is an immersed minimal surface in $R^{n}$ of finite topological type and the volume growth for extrinsic balls is at most quadratic, then $M$ must have finite total curvature. This is a special case of our theorem. Indeed, a minimal surface necessarily has non-positive Gaussian curvature and the extrinsic distance is dominated from above by the intrinsic distance, hence $M$ satisfies the hypotheses of our theorem and must have finite total curvature.

Theorem. Let $M^{2}$ be a complete, non-compact surface with finite topological type. If $M$ has at most quadratic area growth and the Gaussian curvature of $M$ is either non-negative or non-positive, near infinity of each end, then $M$ must have finite total curvature.

Proof. The fact that $M$ has finite topological type implies that $M$ is diffeomorphic to a compact Riemann surface with finite punctures. In fact, a neighborhood of each puncture corresponds to an end of $M$. Obviously, the theorem follows if we can show that $|K|$ is integrable at each end.

Since each end is diffeomorphic to the punctured disk which is also diffeomorphic to $\mathbb{R}^{2} \backslash \mathbb{D}^{2}$, we represent an end by $\mathbb{R}^{2} \backslash \mathbb{D}^{2}$ with a metric that is complete at infinity. By extending the metric arbitrarily, we may assume that $M$ is diffeomorphic to $\mathbb{R}^{2}$ and the curvature is either non-positive or non-negative on $\mathbb{R}^{2} \backslash \mathbb{D}^{2}$. 
For the case when the Gaussian curvature of $M$ is non-negative near infinity, the surface satisfies

$$
\int_{M} K_{-}<\infty
$$

and Huber's theorem implies that $\int_{M}|K|<\infty$. Hence we only need to prove the theorem for the case when $K$ is non-positive near infinity.

Let us first assume that there exists a simple, closed, $C^{1,1}$ curve $\gamma$, in $\overline{\mathbb{R}^{2} \backslash \mathbb{D}^{2}}$, homotopic to $\mathbb{S}^{1}=\partial \mathbb{D}^{2}$ with non-negative geodesic curvature with respect to the outward normal $\nu$. Note that a $C^{1,1}$ curve is $C^{2}$ almost everywhere. Hence geodesic curvature is defined almost everywhere. In particular, the following argument which involves integrating the geodesic curvature is valid. Then nonpositivity of $K$ on $\mathbb{R}^{2} \backslash \mathbb{D}^{2}$ implies that the curve $\gamma$ has no focal point in the direction of $\nu$. If $\Omega$ denotes the domain bounded by $\gamma$ and $E=\mathbb{R}^{2} \backslash \Omega$ denotes the exterior domain, then we can parametrize $E$ using Fermi coordinates with respect to $\gamma$. In particular, if we take $\theta \in[0,2 \pi]$ to be a parametrization of $\gamma$ which is proportional to arc-length, then each point $x \in E$ can be written as $(\theta, r)$ where $r$ is the distance from $x$ to $\gamma$ and $r=d(\gamma(\theta), x)$. Also, each coordinate curve $\eta(r)$ given by points in $E$ which are at distance $r$ from $\gamma$ is smooth. Let $C(r)$ denotes the domain bounded by $\eta(r)$. Applying the Gauss-Bonnet theorem on $C(r)$, we have

$$
2 \pi=\int_{C(r)} K d A+\int_{\eta(r)} k_{g}
$$

where $k_{g}$ is the geodesic curvature of $\eta(r)$ with respect to the outward pointing normal $\frac{\partial}{\partial r}$. The smoothness of $\eta(r)$ and the first variation formula imply that

$$
\begin{aligned}
\int_{\eta(r)} k_{g} & =\frac{\partial l(\eta(r))}{\partial r} \\
& =\frac{\partial^{2} A(C(r))}{\partial r^{2}},
\end{aligned}
$$

where $l(\eta(r))$ and $A(C(r))$ are the length of $\eta(r)$ and the area of $C(r)$, respectively. If $d s^{2}$ has infinite total curvature, (2) and (3) imply that $\frac{\partial^{2} A(C(r))}{\partial r^{2}}$ increases monotonically to infinity for sufficiently large $r$ as $r \rightarrow \infty$. This contradicts the assumption that $d s^{2}$ has at most quadratic area growth.

We now assume that $M$ does not admit a $C^{1,1}$ curve $\gamma$ in $e=\overline{\mathbb{R}^{2} \backslash \mathbb{D}^{2}}$ which has non-negative geodesic curvature with respect to the outward normal. Let us consider the truncated end $e(R)=e \cap B(R)$ where $B(R)$ is the geodesic ball of radius $R$ with respect to $d s^{2}$ centered at the origin. Let $\beta>0$ be a fixed constant such that $\mathbb{D}^{2} \subset B(\beta)$. For $R>\beta$ sufficiently large, the truncated end $e(R)$ is homeomorphic to a cylinder. Let us consider a curve $\mu$ in $\overline{e(R)}$ which minimizes length among all curves homotopic to $\mathbb{S}^{1}=\partial \mathbb{D}^{2}$. If $\mu$ lies completely in the interior of $e(R)$, then the first variation formula for arc-length asserts that $\mu$ is a 
geodesic. This violates the non-existence assumption of $\gamma$. On the other hand, if $\mu$ intersects both boundaries $\mathbb{S}^{1}$ and $\partial B(R) \cap e$, of $e(R)$, then the length of $\mu$ must be at least twice the distance $d\left(\mathbb{S}^{1}, \partial B(R) \cap e\right)$ from $\mathbb{S}^{1}$ to $\partial B(R)$. However, since $d\left(\mathbb{S}^{1}, \partial B(R) \cap e\right) \geq(R-\beta)$, we have $l(u) \geq 2(R-\beta)$. By taking

$$
R>2 \beta+2 \alpha,
$$

where $2 \alpha=l\left(\mathbb{S}^{1}\right)$, we conclude that $l(\mu)>2 \alpha$. This contradicts the minimizing property of $\mu$. Therefore, $\mu$ must either intersect $\mathbb{S}^{1}$ or $\partial B(R) \cap e$, but not both.

If $\mu \cap \mathbb{S}^{1}=\emptyset$, then the first variation formula implies that $\mu \backslash \mathbb{S}^{1}$ is a geodesic. Also, it was proved in [M-S] that $\mu$ must be $C^{1,1}$. Moreover, it was established in $[\mathrm{A}-\mathrm{B}-\mathrm{B}]$ that the geodesic curvature is non-negative everywhere with respect to the outward normal. This again violates the assumption that $\gamma$ does not exist. Hence $\mu$ must intersect $\partial B(R) \cap e$ non-trivially for all $2 R>2 \beta+2 \alpha$.

If $\partial B(R)$ is smooth, the previous argument asserts that $\mu$ is $C^{1,1}$ and has non-positive geodesic curvature with respect to the outward normal. Since the $l(\mu) \leq 2 \alpha$, the curve $\mu$ must lie in the annulus $(B(R) \backslash B(R-\alpha)) \cap e$. Let $\Omega_{\mu}$ denotes the domain bounded by $\mu$. Using Fermi coordinates $(\theta, t)$ issuing from $\mu$ in the direction of the inward normal and using the fact that $e$ has non-positive curvature, we can now parametrize the set $e \cap \Omega_{\mu}$ given by the intersection of $e$ and $\Omega_{\mu}$. Let us define the curve $\mu_{t}=\left\{x \in e \cap \Omega_{\mu} \mid d(x, \mu)=t\right\}$ and the set $\Omega_{\mu}(t)=\left\{x \in e \cap \Omega_{\mu} \mid d(x, \mu) \leq t\right\}$. Observe that the curvature assumption on $e$ and the fact that $\mu$ has non-positive geodesic curvature with respect to the outward normal imply that the curves $\mu_{t}$ are smooth for $t \leq R-\beta-\alpha$. In fact, these are the coordinate curves $(\cdot, t)$ of the Fermi coordinates. Applying the Gauss-Bonnet formula to the domain $\Omega_{\mu} \backslash \Omega_{\mu}(t)$ and using the identity

$$
-\int_{\mu_{t}} k_{g}=\frac{\partial l\left(\mu_{t}\right)}{\partial t}
$$

for all $0<t \leq R-\beta-\alpha$, we have

$$
\frac{\partial l\left(\mu_{t}\right)}{\partial t}=-2 \pi+\int_{\Omega_{\mu} \backslash \Omega_{\mu}(t)} K
$$

Integrating with respect to $t$ from 0 to $\frac{R}{2}-\alpha$, we conclude that

$$
\begin{aligned}
l\left(\mu_{\left(\frac{R}{2}-\alpha\right)}\right)-l(\mu) & =-2 \pi\left(\frac{R}{2}-\alpha\right)+\int_{0}^{\frac{R}{2}-\alpha} \int_{\Omega_{\mu} \backslash \Omega_{\mu}(t)} K \\
& \leq-\left(\frac{R}{2}-\alpha\right)\left(2 \pi-\int_{\Omega_{\mu} \backslash \Omega_{\mu}(t)\left(\frac{R}{2}-\alpha\right)} K\right) .
\end{aligned}
$$

In particular, the minimality of $\mu$ asserts that

$$
l(\partial B(R)) \geq l(\mu) \geq\left(\frac{R}{2}-\alpha\right)\left(2 \pi-\int_{\Omega_{\mu} \backslash \Omega_{\mu}(t)\left(\frac{R}{2}-\alpha\right)} K\right) .
$$


The facts that $\mu \cap \partial B(R) \neq \emptyset$ and $l(\mu) \leq 2 \alpha$ imply that $\mu$ is at distance at most $\alpha$ from $\partial B(R)$. Hence, $\Omega_{\mu} \backslash \Omega_{\mu}(t)\left(\frac{R}{2}-\alpha\right)$ must contain $B\left(\frac{R}{2}\right)$. Inequality (5) implies that

$$
R^{-1} l(\partial B(R)) \geq R^{-1}(R / 2-\alpha)\left(2 \pi-\int_{B(R / 2)} K\right) .
$$

If the integral of $-K$ is infinite, this asserts that $R^{-1} l(\partial B(R)) \rightarrow \infty$, which contradicts the assumption that the area growth is at most quadratic.

To overcome the possibility that $\partial B(R)$ might not be smooth, we simply consider a smooth approximation of the distance $r$ by $\tilde{r}$ satisfying

$$
(1-\epsilon) \tilde{r} \leq r \leq(1+\epsilon) \tilde{r}
$$

and apply the previous argument to $\tilde{r}$. This completes the proof of the theorem.

\section{References}

[A-B-B] S. Alexander, I. D. Berg and R. L. Bishop, The Riemannian obstacle problem, Ill. J. Math. 31 (1987), 167-184.

[C] Q. Chen, On the total curvature and area growth of minimal surface in $R^{n}$, preprint.

[CV] S. Cohn-Vossen, Kürzeste Wege and Totalkrümmung auf Flächen, Compositio Math. 2 (1935), 69-133.

[F] R. Finn, On a class of conformal metrics with application to differential geometry in the large, Comm. Math. Helv. 40 (1965), 1-30.

[H] P. Hartman, Geodesic parallel coordinates in the large, Amer. J. Math. 86 (1964), 705-727.

[Hu1] A. Huber, On subharmonic functions and differential geometry in the large, Comm. Math. Helv. 32 (1957), 13-72.

[Hu2] A. Huber, Vollständige konforme Metriken und isolierte Singularitäten subharmonischer Funktionen, Comm. Math. Helv. 41 (1966), 105-136.

[L-T] P. Li and L. F. Tam, Complete surfaces with finite total curvature, J. Diff. Geom. 33 (1991), 139-168.

[M-S] A. Marino and D. Scolozzi, Geodetiche con ostacolo, Boll. Un. Mat. Ital. B (6) 2 (1983), 1-31.

[S] K. Shiohama, Total curvature and minimal area of complete open surfaces, Proc. AMS 94 (1985), 310-316.

Peter Li

Department of Mathematics

University of California, Irvine

Irvine, CA 92697-3875

USA

e-mail: pli@math.uci.edu

(Received: October 16, 1995) 